\title{
Editorial: Trends and Advances in Nano-Structured Materials With Novel Structures and Properties
}

\begin{abstract}
Yanzhong Tian ${ }^{1,2 *}$, Zhefeng Zhang $^{3,4 *}$ and Nobuhiro Tsuji ${ }^{5,6 *}$
${ }^{1}$ Key Laboratory for Anisotropy and Texture of Materials (Ministry of Education), School of Materials Science and Engineering, Northeastern University, Shenyang, China, ${ }^{2}$ Research Center for Metallic Wires, Northeastern University, Shenyang, China, ${ }^{3}$ Laboratory of Fatigue and Fracture for Materials, Shi-changxu Innovation Center for Advanced Materials, Institute of Metal Research, Chinese Academy of Sciences, Shenyang, China, ${ }^{4}$ School of Materials Science and Engineering, University of Science and Technology of China, Hefei, China, ${ }^{5}$ Department of Materials Science and Engineering, Kyoto University, Kyoto, Japan, ${ }^{6}$ Elements Strategy Initiative for Structural Materials (ESISM), Kyoto University, Kyoto, Japan
\end{abstract}

Keywords: strength, ductility, ultrafine grain, recrystallization, nanostructure, high-entropy alloy

\section{Editorial on the Research Topic}

Trends and Advances in Nano-structured Materials with Novel Structures and Properties

The research topic "Trends and Advances in Nano-structured Materials with Novel Structures and Properties" was organized to collect recent advances of novel structural metallic materials with superior mechanical properties.

Advanced metallic materials with nanostructures show promising mechanical properties. For realizing advanced structural materials, it is critical to overcome the strength-ductility trade-off or optimize them concurrently (Tsuji et al., 2019). There have been significant breakthroughs in developing novel nanostructures in the last decade ( $\mathrm{Wu}$ and $\mathrm{Zhu}, 2017)$. For example, fully recrystallized nanostructures, heterogeneous nanostructures and gradient nanostructures have been developed in various metallic systems, which exhibit excellent tensile properties and fatigue properties. The nanostructured metallic materials with recrystallized structure can even manifest better fatigue strength than the coarse-grained and plastically deformed nanocrystalline counterparts (Liu et al., 2018), indicating that it is crucial to carefully design the suitable microstructure for superior mechanical performances. The materials having recrystallized nanostructures can also exhibit unconventional mechanical behavior in contrast to the coarse-grained counterparts. For example, apparent yield-drop phenomenon and extensive Lüders deformation were often detected during tensile test (Gao et al., 2019).

Bulk nanostructured materials can be prepared via various top-down techniques based on intense plastic straining. Furthermore, dynamic or static heat treatments were applied to reconstruct the microstructures for optimized properties. In this research topic, cutting-edge advances of steels and high-entropy alloys (HEAs) were introduced. In contrast to the conventional powder metallurgy strategy which has some inherent disadvantages, two-phase oxide dispersion strengthened steels were processed by casting and hot rolling (Wang et al.). In this way, a heterogeneous microstructure consisting of ferritic and martensitic lamellae was achieved, providing a possible way to obtain superior mechanical properties. Meanwhile, a single-phase austenitic Fe-30Mn-0.14C-7Cr- $0.26 \mathrm{Ni}$ steel was processed by cold rolling and annealing to realize heterogeneous and fully recrystallized nanostructures (Chen et al.). A new Hall-Petch relationship and extra-hardening were revealed in the nano-regime, which can be related to the limited dislocation sources.

HEAs represent novel materials design strategies and have attracted worldwide attentions due to their superior mechanical and functional properties. Similar to conventional metallic materials, nanostructured HEAs also exhibit promising properties. For example, the well-known CoCrFeMnNi Cantor alloy can be processed by simple cold rolling and annealing treatments to achieve 
recrystallized nanostructures and balanced strength-ductility synergy at cryogenic temperatures (Sun et al., 2018). In this research topic, new advances of HEAs have been included. Three low-density refractory NbTiVZr, $\mathrm{Nb}_{0.5} \mathrm{TiVZr}$, and $\mathrm{NbTiV}$ alloys were developed, and the high-temperature compression properties were investigated (Jia et al.). In contrast to the well-known Inconel 718 alloy, the NbTiVZr alloy can exhibit better yield strength at temperatures higher than $1,073 \mathrm{~K}$. Meanwhile, eutectic HEAs have been widely studied among the HEA family. In contrast to the other HEAs, the casted eutectic HEAs generally possess higher yield strength which is attributed to the fine nanostructures formed through alternating distribution of two phases under solidification. Eutectic $\mathrm{AlCoCrFeNi}_{2.1}$ and hyper-eutectic $\mathrm{AlCo}_{0.4} \mathrm{CrFeNi}_{2.7}$ were casted, and they showed similar yield strength of $540 \mathrm{MPa}$. However, the $\mathrm{AlCo}_{0.4} \mathrm{CrFeNi}_{2.7} \mathrm{HEA}$ possessed much higher impact toughness than the eutectic AlCoCrFeNi $i_{2.1}$, providing an accessible way to improve the strength and toughness simultaneously (Zhang and Zhang). In addition, conventional thermal mechanical processes were applied to produce bulk specimens with nanostructures in $\mathrm{Ti}_{35} \mathrm{Zr}_{27.5} \mathrm{Hf}_{27.5} \mathrm{Nb}_{5} \mathrm{Ta}_{5}$ and $\mathrm{Ti}_{35} \mathrm{Zr}_{26} \mathrm{Hf}_{26} \mathrm{Nb}_{6.5} \mathrm{Ta}_{6.5}$ HEAs (Guillot et al.). Tensile properties could be controlled by changing volume fractions of two phases and inherent grain

\section{REFERENCES}

Gao, S., Bai, Y., Zheng, R., Tian, Y., Mao, W., Shibata, A., et al. (2019). Mechanism of huge Lüders-type deformation in ultrafine grained austenitic stainless steel. Scr. Mater. 159, 28-32. doi:10.1016/j.scriptamat. 2018.09.007

Liu, R., Tian, Y. Z., Zhang, Z. J., Zhang, P., An, X. H., and Zhang, Z. F. (2018). Exploring the fatigue strength improvement of Cu-Al alloys. Acta Mater. 144, 613-626. doi:10.1016/j.actamat.2017.11.019

Sun, S. J., Tian, Y. Z., An, X. H., Lin, H. R., Wang, J. W., and Zhang, Z. F. (2018). Ultrahigh cryogenic strength and exceptional ductility in ultrafine-grained CoCrFeMnNi high-entropy alloy with fully recrystallized structure. Mater. Today Nano. 4, 46-53. doi:10.1016/j.mtnano.2018.12.002

Tsuji, N., Gholizadeh, R., Ueji, R., Kamikawa, N., Zhao, L., Tian, Y., et al. (2019). Formation mechanism of ultrafine grained microstructures: various sizes, and ultrahigh yield strength up to $1 \mathrm{GPa}$ could be achieved in these two HEAs. This work has introduced an elaborative strategy to introduce heterogeneities and nanostructures concurrently in two-phase HEAs.

We hope that the papers in the present Research Topic can stimulate new exciting research work in the advances of metallic materials with novel structures and properties.

\section{AUTHOR CONTRIBUTIONS}

All authors listed have made a substantial, direct, and intellectual contribution to the work, and approved it for publication.

\section{FUNDING}

This work was supported by the Fundamental Research Funds for the Central Universities under grant No. N180204015. YT, ZZ, and NT were supported by Chinese Academy of Sciences (CAS) and Japan Society for the Promotion of Science (JSPS) through the Bilateral Program (No. GJHZ1774). All the supports are gratefully acknowledged.

possibilities for fabricating bulk nanostructured metals and alloys. Mater. Trans. 60, 1518-1532. doi:10.2320/matertrans.MF201936

Wu, X., and Zhu, Y. (2017). Heterogeneous materials: a new class of materials with unprecedented mechanical properties. Mater. Res. Lett. 5, 527-532. doi:10. $1080 / 21663831.2017 .1343208$

Conflict of Interest: The authors declare that the research was conducted in the absence of any commercial or financial relationships that could be construed as a potential conflict of interest.

Copyright (C) 2020 Tian, Zhang and Tsuji. This is an open-access article distributed under the terms of the Creative Commons Attribution License (CC BY). The use, distribution or reproduction in other forums is permitted, provided the original author(s) and the copyright owner(s) are credited and that the original publication in this journal is cited, in accordance with accepted academic practice. No use, distribution or reproduction is permitted which does not comply with these terms. 